\title{
Truth-aware Optimal Decision-making Framework with Driver Preferences for V2V Communications
}

\author{
Mingshun Sun* Ming Li* Ryan Gerdes ${ }^{\dagger}$ \\ ${ }^{*}$ Department of ECE, The University of Arizona, Tucson, AZ \\ ${ }^{\dagger}$ Department of ECE, Virginia Tech, Arlington, VA \\ Email: *\{mingshunsun,lim\}@email.arizona.edu, ${ }^{\dagger}$ rgerdes@ vt.edu
}

\begin{abstract}
In Vehicle-to-Vehicle (V2V) communications, malicious actors may spread false information to undermine the safety and efficiency of the vehicular traffic stream. Thus, vehicles must determine how to respond to the contents of messages which maybe false even though they are authenticated in the sense that receivers can verify contents were not tampered with and originated from a verifiable transmitter. Existing solutions to find appropriate actions are inadequate since they separately address trust and decision, require the honest majority (more honest ones than malicious), and do not incorporate driver preferences in the decision-making process. In this work, we propose a novel trust-aware decision-making framework without requiring an honest majority. It securely determines the likelihood of reported road events despite the presence of false data, and consequently provides the optimal decision for the vehicles. The basic idea of our framework is to leverage the implied effect of the road event to verify the consistency between each vehicle's reported data and actual behavior, and determine the data trustworthiness and event belief by integrating the Bayes' rule and Dempster Shafer Theory. The resulting belief serves as inputs to a utility maximization framework focusing on both safety and efficiency. This framework considers the two basic necessities of the Intelligent Transportation System and also incorporates drivers' preferences to decide the optimal action. Simulation results show the robustness of our framework under the multiple-vehicle attack, and different balances between safety and efficiency can be achieved via selecting appropriate human preference factors based on the driver's risk-taking willingness.
\end{abstract}

\section{INTRODUCTION}

Connected vehicles rely on Vehicle-to-Vehicle (V2V) communications to exchange information such as vehicle states and road conditions with each other, forming an Intelligent Transportation System (ITS) [1]. Such V2V information can provide guidance beyond the 'line of sight' and help vehicles perceive potential dangers in an extended space and time horizon [2]. Additionally, recent advancements in autonomous (or semi-autonomous) vehicle technology depend on $\mathrm{V} 2 \mathrm{~V}$ communication to achieve higher travel safety and efficiency [3]. In a typical cooperative collision avoidance application of cooperative adaptive cruise control (CACC), if the leading vehicle observes a collision event ahead, it would broadcast this event along with its regular messages and then all the following vehicles will decelerate simultaneously [4].

However, connected vehicles are expose to various security threats. Hackers can compromise vehicles remotely and broadcast messages about fake traffic conditions (e.g.,

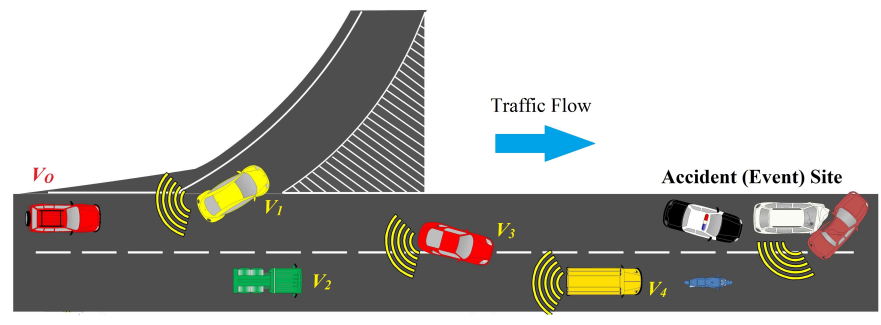

Fig. 1. A Simple Motivating Example.

accident where none has occurred), so as to induce serious consequences. Relevant real-world incidents include the Google/Waze hacks [5]-[7] whereby false traffic information would lead users of the service to believe traffic congestion was present when it was not. As a result, those vehicles will slow down or change their routes, which would cause congestion and greatly reduce the road capacity. We will use a simple example to illustrate it next.

\section{A. Motivating Example}

Fig. 1 shows a two-lane highway where vehicles form a local Vehicular Ad Hoc Network (VANET) using V2V communications. Suppose that an accident happens ahead of vehicle $V_{0}$ (which it cannot observe directly). An honest neighboring vehicle $V_{3}$ broadcasts a message which says 'An accident happens ahead and I'm switching to another lane'. If this is the only message $V_{0}$ receives, it would start braking early to avoid crashing into the accident site. However, suppose that hackers remotely compromise two other neighboring vehicles $V_{1}$ and $V_{2}$, and make them maliciously broadcast false information such as 'there is no accident ahead and I'm accelerating'. Now $V_{0}$ cannot distinguish the existence of this accident. Believing in the majority and not decelerating may ultimately lead to crash. Thus, it is critical for vehicles to assess the truthfulness of $\mathrm{V} 2 \mathrm{~V}$ messages and respond in an optimal manner.

\section{B. Challenges and Our Contributions}

Many challenges arise when designing a truth-aware decision-making framework in a V2V environment, that is robust to false message injection attacks. First, since attackers can report a fake accident or hide an actual event at any time, vehicles must be able to assess the trustworthiness of each report and choose the optimal action in real time. This renders 
previous approaches that leverage node/entity trust inapplicable [8], [9], since they need a long time to establish trust, and an attacker could suddenly lie after gaining such trust. Besides, multiple vehicles in the local neighborhood may be hacked simultaneously (or attackers can be equipped with multiple transmitters), which will break the honest majority assumption adopted by most previous works [10], [11].

Since vehicles communicate in a peer-to-peer fashion, local information gathered could be highly incomplete, dynamic and uncertain. Also, each vehicle as a fusion center must deal with such uncertainties when making its decision, let alone that part or even majority of the data can be intentionally malicious. We're concerned with how a vehicle should react to a message when its belief in the truthfulness of the message is uncertain. Moreover, vehicle drivers have different preferences on how ambiguous data should be reacted to. For example, some of them are aggressive while others are more conservative. Such human factors should be considered when making driving decisions under uncertain information. But it does not necessarily violate the 'autonomous' definition since no more operation is mandatorily required from the driver. Ideally, only an interface should be provided for a driver to reflect his/her own riskseeking or risk-aversion preference.

To cope with the above challenges, we propose a novel truthaware decision framework, which exploits the verification of a vehicle's implied effect of its report to assess the report's truthfulness. That is, whether its actual behavior matches with its supposed action assuming an event's existence/non-existence (e.g., if $V_{3}$ claims there is an accident ahead, we should observe that it decelerates or switches to another lane). More specifically, when a vehicle receives potentially conflicting messages about an event from multiple neighboring vehicles, it evaluates both the messages and observed actions of its neighbors to obtain each message's trustworthiness based on the implied effect, and ultimately form a robust belief about the event. We achieve this through a novel combination of Bayes' rule and Dempster Shafer Theory (DST).

In addition, in order to provide an optimal response to uncertain information, we propose to let vehicles act provisionally in the beginning when the belief about an event is small (since we are concerned with events that vehicles cannot directly observe). Eventually, as more evidence arrives, we gradually know the true state of the world (i.e., whether the incident occurred or not) so our belief in an event will eventually converge to 1 or 0 which means the event happens with probability 1 or 0$)$. Moreover, we incorporate driver preference into the decision-making process, by allowing drivers to choose their respective risk-taking levels. A preference decision function that combines driver preferences and the event belief would output the user's preference on safety and efficiency, which consequently becomes the inputs to a utility maximization problem and outputs the optimal action that the driver should take (e.g., deceleration amount).

In summary, our main contributions in this work include:

- We present the first framework that leverages implied effect to estimate the truthfulness of an event in the $\mathrm{V} 2 \mathrm{~V}$ setting, which provides robust data trustworthiness assessment even under a dishonest majority scenario.

- A novel combination of Bayes rule and DST is proposed to estimate and update the belief in a reported event, which can deal well with uncertainty and conflicts (including false data) within different sources of evidence.

- We propose an optimal, dynamic truth-aware decisionmaking framework for $\mathrm{V} 2 \mathrm{~V}$ communications, which is formulated as a utility-maximization problem where the utility function reflects a balance between the vehicle safety and efficiency. To the best of our knowledge, it is the first framework that takes driver preferences into account in the decision-making under data uncertainty.

Finally, simulation results demonstrate that our framework is able to achieve better safety-efficiency trade-off than previous data trust assessment approaches, under various attack scenarios. We also show that drivers could flexibly choose their respective risk-taking factors to ensure a certain level of safety.

\section{RELATED WORK}

Existing work in trust and decision making in VANETs mainly focused on entity trust and reputation management, data-centric trust, and misbehavior detection.

\section{A. Entity-based Trust Framework in VANETs}

Early works focused on assessing vehicles' trustworthiness at the entity level [8], [9], [12]. These frameworks require a lengthy interaction and a large amount of historical data for reputation assessment. The long-term trust can be exploited by attackers that broadcast false claims in a short-term to remain undetected. Thus, a data-centric trust framework is needed.

\section{B. Data-based Trust and Misbehavior Detection in VANETs}

An early work in ephemeral trust was done by Raya et. al. [10], where one vehicle combines different sources of evidence by data fusion techniques such as DST or majority voting to assess the trust of each piece of information from other vehicles. Ghosh et. al. [13] used consistency-check among a vehicle's own reports to aids the detection of false alarm messages. However, attackers are able to modify their reported claims and force them consistent to avoid inconformity checking. Wei et. al. [14] proposed a trust-based mechanism to establish trust with direct interactions and recommendations, which could provide extra protection from inside attackers. However, above work rely on the vehicles' trust to combine their reports, thus they require an honest majority of vehicles to be secure. In [15], we proposed a false vehicle data filtering and secure vehicle tracking framework for $\mathrm{V} 2 \mathrm{~V}$ communications based on secure physical layer sensing without assuming an honest majority. However, it does not address event truthfulness and decision.

\section{Decision-making Framework in VANETs}

Early work on decision-making such as Coussement et. al. [16] proposed a decision-support protocol based on the intrusion detection system installed on both vehicle and RSU 
to broadcast the attack information towards the close vehicular clusters. Zhang et. al. [17] designed a trust-based action selection model, which propagates peers' opinions to choose the proper action such as 'following' or 'not following'. Above frameworks do not consider the basic requirements (safety and efficiency) of ITS when making decisions and mainly rely on the information comparison. But they need honest majority as a prerequisite. Thus, an honest majority is a crucial assumption in these works and also they do not consider user preferences in their decision-making process.

In summary, a truth-aware decision-making framework that can estimate the message truthfulness (without assuming honest majority) and also take into account the driver preferences when making decisions is missing from the literature.

\section{Problem Statement}

\section{A. System Model and Assumptions}

Primarily, we assume that all vehicles are equipped with a common radio interface, which communicates with each other using a standard V2V protocol, such as DSRC [18] or WAVE [1]. Per the communication protocols in these standards, vehicles broadcast their internal state information including position, velocity, and acceleration (PVA) periodically (about 10 messages/second), which can be obtained via their GPS or vehicle motion sensors. Upon perceiving a safety-related event either by receiving a message from the source or directly observing it via sensors (such as an accident, disabled car, congestion), vehicles will broadcast event-triggered warning messages, along with the event location/lane information and its own belief regarding the event $E$ (reported belief about $E$ ). Our framework is applicable to both active and passive event source which differs in terms of whether actively broadcasting safety-related messages or not.

We also assume that vehicles only report their own beliefs (rather than the combined belief from other vehicles' reports), that would avoid the inter-message redundancy. Although this limits reporting vehicles to be within the one-hop range of the event, their messages are still useful for vehicles behind them that cannot directly observe the event (the wireless communication range in DSRC can be up to 1000 meters). Event belief propagation and assessment in a multi-hop message forwarding scenario will be our future work.

\section{B. Threat Model}

We consider an active attacker (which could take control of one or multiple vehicles on the road) that injects false data in the broadcast messages, who can falsify both event reports and the PVA data. Note that, our attack model is general in the sense that it can include multiple colluding attackers as well. We assume the communication protocols are secured (e.g., vehicles are loaded with private/public key pairs that certify their digital identity), such that entity authentication and message integrity can be guaranteed by traditional cryptographic means. Besides, we assume that vehicles can securely detect false motion states (PVA) or track the states of other neighboring vehicles (with certain accuracy level).
This can be achieved either using on-board sensors such as radar/lidar/cameras [19]-[21], or by sensing wireless physical layer properties (e.g., secure Doppler shift, angle-of-arrival which are used in our previous work [15]). To make the false event report and its own behavior consistent with each other, an attacker can also falsify its own PVA data. For example, if it reports there is an accident ahead but in fact there is not, it will also report braking and reducing speed while in fact it is not. However, since we assume false PVA data can be detected using traditional approaches, we can observe the vehicle's real movement regardless of whether it falsifies its PVA.

Besides, we do not assume an honest majority of vehicles in any local neighborhood. In fact, in the worst case, all neighboring vehicles can be malicious. Note that, we do not consider denial-of-service attacks in this paper. While the availability of $\mathrm{V} 2 \mathrm{~V}$ messages is also important, when all the messages are jammed, vehicles may choose to fall back to traditional operation modes without $\mathrm{V} 2 \mathrm{~V}$ communications (purely rely on sensors [22]).

Finally, our goal in this paper is to enable each individual vehicle to derive an 'event belief' based on other vehicles' reports, its own sensing results of others' movement (or direct observation of the event if it has), and the nature of the event (which determines the implied effect). In addition, the vehicle needs to compute optimal actions in response to such a belief.

\section{PRELIMINARIES}

In this section, several techniques are introduced and used as building blocks in our proposed framework.

\section{A. Demspter-Shafer Theory (DST)}

Dempster-Shafer theory is effective to evaluate the belief and plausibility of an event by combining different sources of evidence, especially under the existence of data uncertainty and conflicts. It has been adopted in a wide range of applications such as data fusion [23] and decision-making [10].

1) Dempster-Shafer Evidence Theory: In DST, the elements in the frame of discernment $\Theta$ are mutually exclusive. It defines $m: 2^{\Theta} \rightarrow[0,1]$ as the Basic Probability Assignment (BPA) which satisfies

$$
\Sigma_{A \subseteq \Theta} m(A)=1 ; m(\emptyset)=0,
$$

where $A$ is a subset of the power set. If $m(A)>0, A$ is called a focal element. When there exists multiple independent evidences, the combined belief in $A$ can be expressed based on the Dempster's rule of combination as follows:

$$
\begin{gathered}
m_{12}(A)=\frac{\sum_{B \cap C=A} m_{1}(B) m_{2}(C)}{1-K} \\
K=\sum_{B \cap C=\emptyset} m_{1}(B) m_{2}(C)
\end{gathered}
$$

$K$ represents the total conflicting or contradictory mass assignments. For Dempster rule of combination, $1-K$ is the normalization factor which ignores the conflicts and attributes their related BPA to the empty set. Dempster's rule of combination 
TABLE I

DIFFERENT EVIDENCE COMBINATION RULES FOR DST

\begin{tabular}{|c|c|}
\hline Name & Definition \\
\hline $\begin{array}{l}\text { Yagers' Rule } \\
\text { [24] }\end{array}$ & $m_{12}(A)=\sum_{B \cap C=A} m_{1}(B) m_{2}(C)$ \\
\hline $\begin{array}{l}\text { Zhang's } \\
\text { Rule [25] }\end{array}$ & $m_{12}(A)=\frac{\sum_{B \cap C=A} \frac{|A|}{|B||C|} m_{1}(B) m_{2}(C)}{1-\sum_{B \cap C=\emptyset} m_{1}(B) m_{2}(C)}$ \\
\hline $\begin{array}{l}\text { Weighted } \\
\text { DST [26] }\end{array}$ & $m_{12}(A)=\frac{\sum_{B \cap C=A}\left[w_{1} m_{1}(B) w_{2} m_{2}(C)\right]}{1-\sum_{B \cap C=\emptyset}\left[w_{1} m_{1}(B) w_{2} m_{2}(C)\right]}$ \\
\hline $\begin{array}{ll}\text { Han's } & \text { Rule } \\
{[23]} & \end{array}$ & $\begin{array}{l}m_{12}(A)=\sum_{\substack{B \cap C=A \\
m_{2}(D)}} m_{1}(B) m_{2}(C) \\
\sum_{A \cap D=\emptyset} \omega_{1} m_{1}(A) \omega_{2} m_{1}(E) m_{2}(A) \\
A \cap E=\emptyset\end{array}$ \\
\hline
\end{tabular}

is based on the assumption that all sources of evidence have the same accountability. In other words, it is symmetric. When highly conflicted evidence occurs, the above method results in some counter-intuitive results [24]. Besides, since the source can be unreliable or untrustworthy due to the noise or malicious attacks, we should assign weights for different sources.

2) Other Combination Rules: In light of the drawbacks of basic DST rules, many other combination rules have been proposed to address conflicting evidence. Several popular methods are listed and introduced in table I.

The Yager's rule attributes the conflicting assignment to the universal set rather than any subset, which leads to a more conservative result than Dempster's rule. Besides, Zhang's Rule introduces a measure of an intersection of two sets based on cardinality. These two approaches cannot deal with the conflicting evidence as well as highly unreliable sources. The weighted DST takes the reliability in sources into consideration where $w_{1}$ and $w_{2}$ are the weight (trust/reliability) of two evidence sources. It adopts the reliability of both Basic Probability Assignment (BPA) and the normalization factor. But if one of these sources has a lower weight, the combination result will suffer a large loss. As for Han's approach, it is a better choice for our problem (we will explain in Sec. V).

\section{B. Utility Functions}

In economics, the utility function is an important concept that measures the preference over a set of goods and services. This concept is an important underpinning of rational choice theory, which is able to leverage different potential choices [27]. Mathematically, utility functions provide a mapping between the actual measure of money and the perceived value of money in economics. They need to be measurable functions on the probability space. A utility function is an increasing function because it is assumed that everyone would value more money over less money. Here we list several most common used utility functions [28]:

- Exponential function: $U(x)=e^{x}$

- Log function: $U(x)=\log x$

- Power function: $U(x)=\frac{x^{\alpha}-1}{\gamma}$, where $\gamma<1$

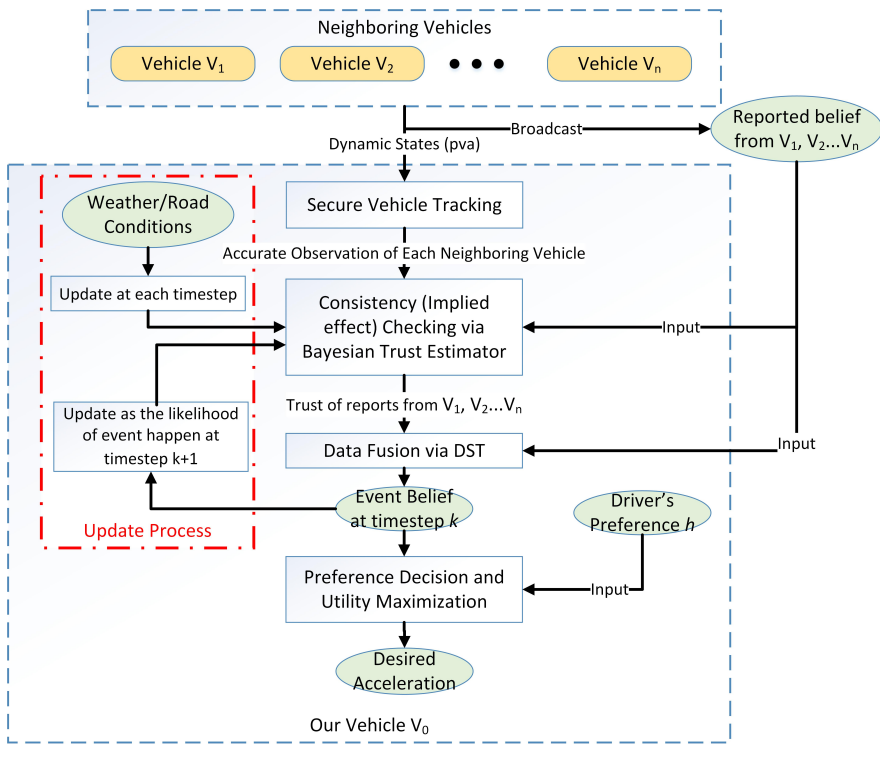

Fig. 2. Proposed Framework

where $x$ represents the money and $U$ is the utility. In this paper, what we want to measure is not money, but the traffic safety and efficiency, which are the two most important goals that drivers would like to achieve on the road. Thus, utility functions for these two goals should be carefully chosen to better represent drivers' real-world preferences.

\section{Proposed FrameWORK}

In this section, we present the proposed framework to achieve message truthfulness estimation, event belief formation and optimal vehicle response in $\mathrm{V} 2 \mathrm{~V}$ communication (shown in Fig. 2). The framework runs iteratively in rounds. We introduce Bayes' rule to estimate the posterior trustworthiness of each event report by verifying the consistency between one vehicle's movement and its claim about an event, via its implied effect. To cope with the uncertainty and conflicts in different pieces of evidence, the DST and Han's combination rule is applied to handle multiple vehicles in each round, which treats their message trustworthiness as weights to form a event belief. Then, the updated event belief is fed back to the Bayesian trust estimator in the next round. Afterwards, in order to make an optimal decision based on driver's preference on safety/efficiency under data uncertainty, we incorporate drivers' risk-taking attitude and the event belief into a preference decision function which consequently becomes the inputs to a utility maximization problem and outputs the optimal vehicle acceleration.

\section{A. Bayes' Estimator for Event Report Trustworthiness}

In this paper, we adopt the Bayes' rule as the theoretical foundation to estimate the truthfulness of each vehicle's event report received. We define report trust as the extent that a report is true (i.e., the reported event actually exists). Then we define a safety-related event set $\mathscr{E}=\left(E_{1}, E_{2} \ldots, E_{n}\right)$, which includes the accident, congestion, police/fire emergency, etc. 
Since the existence of an event $E_{j}$ has implied effects on the reporting vehicle's behavior (i.e., movement/motion states), if we observe the desired behavior that is consistent with the event claimed in the vehicle's report, it can corroborate that event. Otherwise, the report is discredited and we can assign a lower trust to it. Intuitively, we can quantify vehicle $V_{i}$ 's report trust $T_{V_{i}}$ as the consistency between $V_{i}$ 's actual movement and its event report, based on the posterior probability of an event $E_{j}$ is true given our vehicle $V_{0}$ 's observations about $V_{i}$. However, a vehicle's report is not binary since it also includes its own belief $b_{i}$ about $E_{j}$, which ranges from 0 to 1 . A larger $b_{i}$ means $V_{i}$ claims a higher confidence about the existence of $E_{j}$. Thus, we will incorporate the reported belief as follows:

$$
T_{V_{i}}=b_{i} \cdot P\left[E_{j} \mid O_{i}\right]+\left(1-b_{i}\right) \cdot P\left[\overline{E_{j}} \mid O_{i}\right]
$$

where $E_{j}$ denotes an event exists, $\overline{E_{j}}$ denotes an event's non-existence. $P\left[\overline{E_{j}}\right]=1-P\left[E_{j}\right]$. Besides, $O_{i}$ represents $V_{i}$ 's behavior that $V_{0}$ has observed. $P\left[E_{j} \mid O_{i}\right]$ is the posterior probability of an event's existence given the behavior of this vehicle. Intuitively, if $V_{i}$ 's behavior supports the existence of $E_{j}$ and $b_{i}$ is also large, we should have more trust in $V_{i}$ 's report. However, if $V_{i}$ reports a low belief of $E_{j}$ 's existence but its behavior indicates the existence of $E_{j}$, we will assign a low trust on its report. Using the Bayes' theorem, we have:

$$
T_{V_{i}}=b_{i} \cdot \frac{P\left[O_{i} \mid E_{j}\right] P\left[E_{j}\right]}{P\left[O_{i}\right]}+\left(1-b_{i}\right) \cdot \frac{P\left[O_{i} \mid \overline{E_{j}}\right] P\left[\overline{E_{j}}\right]}{P\left[O_{i}\right]}
$$

Note that, the prior probability of events (e.g., traffic accidents) can be obtained by transportation surveys or research, such as accident occurrence rate on highways. $T_{V_{i}}$ is meticulously scaled by the $P\left[O_{i}\right]$, which can be decomposed by the law of total probability:

$$
P\left[O_{i}\right]=\sum_{k=1}^{n} P\left[O_{i} \mid F_{k}\right] \cdot P\left[F_{k}\right],
$$

where $F_{k}$ is a set of disjoint factors whose union is the entire possible factor space. Next, we provide some key details to implement and compute the report trust based on the above.

(1) Without loss of generality, we define a tuple (acceleration, speed, and lane-switching state) to represent the observable behavior of a vehicle. Each element in this tuple has two disjoint and complementary states. For example, the acceleration has two states, $a>0.2 \mathrm{~m} / \mathrm{s}^{2}$ or $a<-0.2 \mathrm{~m} / \mathrm{s}^{2}$, since an absolute acceleration lower than $0.2 \mathrm{~m} / \mathrm{s}^{2}$ usually is not caused by drivers but random factors. The speed's two states are $v \geq 5 \mathrm{~m} / \mathrm{s}$ and $v \leq 5 \mathrm{~m} / \mathrm{s}$, since vehicles usually do not drive below $5 \mathrm{~m} / \mathrm{s}$ in normal traffic conditions. The laneswitching state can be either 1 or 0 , where 1 means a vehicle is switching to another lane, 0 means not. Thus, there are 8 possible observations of each vehicle at each time step.

(2) Multiple factors could lead to the same vehicle behavior. For example, vehicles can slow down due to rain or icy road. Thus, we use a tuple to characterize the space of $F_{k}$ : (weather condition, event's existence). We assume weather condition set $\mathscr{W}$ has $l$ weather conditions such as rain, snow, fog, wind, etc, denoted as $W_{l}$. Their prior probabilities can be obtained from radio or weather forecasts. Thus, this tuple has $l \cdot n$ disjoint possibilities. For simplicity, we assume that $W_{l}$ and $E_{j}$ are independent of each other, and then the prior probabilities of each factor tuple can be expressed as a product of probabilities of each element in it. For example, if there are two weather conditions $W_{1}, W_{2}$ and two events $E_{1}, E_{2}$ in $\mathscr{W}$ and $\mathscr{E}$, respectively. Then $P\left[W_{l}, E_{j}\right]=P\left[W_{l}\right] \cdot P\left[E_{j}\right], \forall l, j \in\{1,2\}$.

Once we have the report trust, we can distinguish the truthful or fake reports by applying a proper threshold on it. A report trust value above this threshold may imply a truthful report. Although there is not a perfect threshold for such classification, it would benefit the driver by providing the approximate percentage of dishonest vehicles when choosing the appropriate risk-seeking parameter in Sec. V-C.

\section{B. Data Fusion via DST}

After we get the report trust of each neighboring vehicle, we will combine the reported belief from each vehicle and its report trust to output the final event belief. Since conflicts and uncertainty can exist among these vehicles' reports, we adopt DST along with Han's combination rule [23] to combine all the received reported beliefs:

$$
\begin{gathered}
m_{12}(A)=\sum_{B \cap C=A} m_{1}(B) m_{2}(C)+\Delta(A) \\
\Delta(A)=\sum_{A \cap D=\emptyset} \omega_{1} m_{1}(A) m_{2}(D)+\sum_{A \cap E=\emptyset} \omega_{2} m_{1}(E) m_{2}(A) \\
w_{1}+w_{2}=1 \quad w_{1}, w_{2} \in[0,1]
\end{gathered}
$$

Here, $m_{12}(A)$ is combined belief for event $\mathrm{A}$ from two sources of evidence, $m_{1}$ and $m_{2} . \Delta(A)$ is the conflict when we fuse evidence regarding A. $w_{1}$ and $w_{2}$ are the normalized weights for two sources. The basic idea is to add conflicting mass assignment into the BPA. For composite events in each evidence, [23] provided an example to calculate the belief of one single event. The basic idea is the calculate the BPA and conflict based on the power set. However, the above expression can only deal with two pieces of evidence, we need to extend it to the multiple sources case. If $r$ pieces of evidence exist, with different weights $w_{1}, \ldots, w_{r}$, we first combine the first two pieces of evidence and get $m_{12}$ based on Eq. (7). Then we use $w_{12}=\frac{w_{1}+w_{2}}{2}$ as the weight of the combination result and then calculate the $m_{123}(A)$ according to $w_{12}, w_{3}$ and $m_{3}$. Then the above process is repeated until the last evidence. For single event evidence, which means only two focal elements exist in it, we show that the multiple sources combination is a simple weighted average, the detailed derivation is in the Appendix. Simply speaking, the $T_{V_{i}}$ and $b_{i}$ are going to fill the role of weights and BPA respectively to deduce the overall event trust. Case study: To demonstrate the advantage of the Han's rule, we conduct an example case study, where three sources of evidence are combined via 4 common DST combination rules and corresponding results are compared. we assume the single event evidence. which only contain the BPA in $A$ and $\bar{A}$. Those three sources of evidence have different BPAs and different report trust values as shown in Table II, 
TABLE II

BPA AND TRUST OF THREE SOURCES OF EVIDENCE

\begin{tabular}{cccc}
\hline Source & Trust & $m(A)$ & $m(\bar{A})$ \\
\hline$m_{1}$ & 0.8 & 0.7 & 0.3 \\
$m_{2}$ & 0.9 & 0.8 & 0.2 \\
$m_{3}$ & 0.1 & 0.01 & 0.99 \\
\hline
\end{tabular}

We present the combination results in table IV:

TABLE III

COMBINATION RESULTS

\begin{tabular}{ccc}
\hline Combination Rules: & $m_{12}(A)$ & $m_{123}(A)$ \\
\hline Dempster's rule & 0.9 & 0.084 \\
Yager's rule & 0.56 & 0.0056 \\
Zhang's rule & 0.45 & 0.042 \\
Weighted DST & 0.555 & 0.495 \\
Han's rule & 0.753 & 0.712 \\
\hline
\end{tabular}

From table III, we can see that the first four combination rules are inappropriate when facing large conflicts caused by the source $m_{3}$ and yet it almost overturns the combined belief when all three pieces of evidence are considered. On the other hand, Han's rule can better reflect the correct fusion result which minimizes the influences of the third evidence. Therefore, we adopt Han's rule in our scheme. That is, after we obtain the report trust from the Bayesian trust estimator, we will use the Han's combination rule to fuse different pieces of evidence and update the event belief for the current time step. Then we feed this updated event belief back to the Bayesian estimator as the new prior probability of the event.

\section{Utility based Optimal Decision-Making}

When our vehicle obtains the event belief, it needs to provide an optimal response to the potential event (e.g., output an acceleration $a$ ). First, we define the safety and efficiency in our framework. Safety should be inversely related to the difference between arriving speed and the speed of traffic while passing the accident site of vehicle $V_{0}$, which may reduce to just the arriving speed if the corresponding lane is blocked (e.g., an accident). The more significant the difference is, the more severe consequences of a collision will bring. Efficiency is related to the difference between nominal speed and predicted arriving speed under some acceleration $a$ at accident site when the event actually does not exist. Other factors that can explain the safety and efficiency are not discussed in this section such as inter-vehicle distance, headway time and vehicle density. They can be evaluated in the future together with the implementation of CACC. Specifically, safety $U_{1}(a)$ and efficiency $U_{2}(a)$ are defined based on [29], [30]:

$$
\begin{gathered}
U_{1}(a)=-e^{0.05 \cdot\left(v_{t}-v_{e}\right)} \\
U_{2}(a)=-\log \left(10 \cdot\left(v_{n}-v_{t}\right)\right),
\end{gathered}
$$

where $v_{t}=\sqrt{v_{0}^{2}+2 \cdot a \cdot s}$ is the arriving speed of vehicle $V_{0}$ at accident site if it keeps using acceleration $a$. Also, $v_{0}$ is the current speed of our vehicle $V_{0}, v_{e}$ is the event speed. $v_{n}$ is the nominal (ideal) speed at accident site (if it doesn't exist), $s$ is the current distance to the accident site. Two scaling factors, 0.05 and 10 make these two functions behave at the same order of magnitude. As $v_{t}$ increases, the safety drops exponentially, whereas the efficiency grows at a decreasing rate, which corresponds to the traffic flow nature. Note that, the above utility functions are chosen only for illustration purposes and they can be defined by the driver/manufacturer.

The preference decision function is defined as:

$$
p=1-e^{-h \cdot B_{E}}
$$

where $p$ is the preference on safety, $h$ is the driver-chosen factor, which reflects driver's risk-taking willingness. $B_{E}$ is the event belief we obtain from the previous data fusion module. A larger $h$ results in a larger $p$ if other parameters are fixed. In other words, a larger $h$ represents a more risk-aversion driver.

Then we combine these two utility functions by the additive utility theory [31] and express the total utility as follows:

$$
U(a)=p \cdot U_{1}(a)+(1-p) \cdot U_{2}(a)
$$

where, $U(a)$ is the total utility value. A larger $p$ represents that the driver puts more importance on safety rather than efficiency. Then we can formulate an optimization problem:

$$
\begin{array}{ll}
\max & U(a)=-p \cdot e^{0.05 \cdot\left(\sqrt{v_{0}^{2}+2 \cdot a \cdot s}-v_{E}\right)} \\
& -(1-p) \cdot \log \left(10 \cdot\left(v_{n}-\sqrt{v_{0}^{2}+2 \cdot a \cdot s}\right)\right) \\
\text { s.t. } \quad & v_{0}^{2}+2 a s \geq 0 \\
& v_{0}^{2}+2 a s-v_{n}^{2} \leq 0 \\
& a_{\max } \geq a \geq a_{\min } \\
& 0 \leq v_{0}+a \cdot \Delta t \leq v_{\max }
\end{array}
$$

where $\Delta t$ is the time step, $a_{\max }$ and $a_{\min }$ are the maximum and minimum values of the acceleration respectively. $v_{\max }$ is the speed limit of a highway. The first constraint demonstrates the arrival speed cannot be less than 0 since we do not allow backward driving. The second constraint guarantees the arrival speed cannot be larger than the nominal speed at the event location. The third constraint limits the acceleration range. Finally, the fourth constraint indicates the speed at the next time step cannot exceed the speed limit and also cannot be negative. The KKT condition are applied to solve this nonlinear optimization problem [32]. After obtaining the candidate KKT points, we plug them back into the objective function and get the desired acceleration that maximizes the utility.

\section{EVALUATION}

In this section, we use simulation to evaluate the attacktolerance and robustness of our proposed framework, as well as compare it with previous methods. We implemented the proposed framework in Matlab R2016a, and simulated it in an example three-lane highway VANET as shown in Fig. 3.

The detailed setup in our simulations is as follows. We consider 10 vehicles on a highway Euclidean space all within onehop range. $x$ and $y$ are longitudinal and latitudinal coordinates respectively. We assume that an accident happens at the centerlane involving two colliding cars, which means the accident speed $v_{e}=0 . V_{0}$ is the decision-making vehicle. $V_{1}$ to $V_{9}$ 


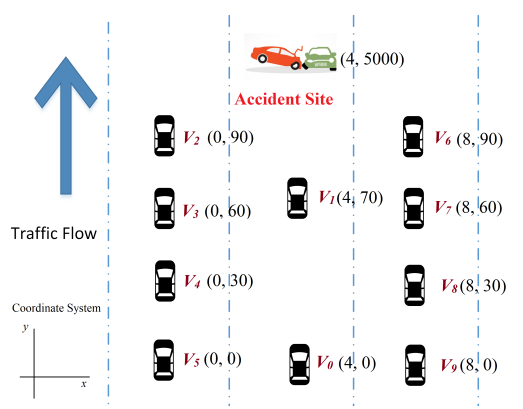

Fig. 3. The simulation setting.

are 9 neighboring vehicles, with different initial positions as shown in Fig. 3. The accident site is 5,000 meters ahead of our vehicle. We assume that $v_{n}=35 \mathrm{~m} / \mathrm{s}$ at the accident site. Besides, the initial speed of all vehicles is $35 \mathrm{~m} / \mathrm{s}$ longitudinal.

For simplicity, we assume a single element (accident) in the event set. The weather condition set only has two complementary elements, 'good weather' and 'bad weather'. Thus, there only remain 4 factor tuples that may affect the neighboring vehicle's movement. $F_{1}$ : (good weather, accident exists), $F_{2}$ : (good weather, accident not exists), $F_{3}$ : (bad weather, accident exists), $F_{4}$ : (bad weather, accident not exists). Then, we predefine the conditional probabilities of all 8 observations given these 4 possible factor tuples by referring [33].

TABLE IV

CONDITIONAL PROBABILITY OF OBSERVATIONS GIVEN FACTOR TUPLES.

\begin{tabular}{cccccccccccc}
\hline \multicolumn{4}{c}{ Observations } & \multicolumn{2}{c}{$P\left[O \mid F_{1}\right]$} & \multicolumn{2}{c}{$P\left[O \mid F_{2}\right]$} & \multicolumn{2}{c}{$P\left[O \mid F_{3}\right]$} & \multicolumn{2}{c}{$P\left[O \mid F_{4}\right]$} \\
swl & $a$ & Speed & EL & NEL & EL & NEL & EL & NEL & EL & NEL \\
\hline 0 & $<-0.2$ & $v<5$ & 0.9 & 0.55 & 0.2 & 0.4 & 0.95 & 0.55 & 0.7 & 0.4 \\
0 & $<-0.2$ & $v \geq 5$ & 0.8 & 0.5 & 0.4 & 0.4 & 0.85 & 0.5 & 0.7 & 0.4 \\
0 & $>0.2$ & $v<5$ & 0.7 & 0.5 & 0.5 & 0.4 & 0.75 & 0.5 & 0.7 & 0.4 \\
0 & $>0.2$ & $v \geq 5$ & 0.2 & 0.4 & 0.8 & 0.7 & 0.1 & 0.4 & 0.3 & 0.7 \\
1 & $<-0.2$ & $v<5$ & 0.9 & 0.1 & 0.3 & 0.3 & 0.7 & 0.2 & 0.6 & 0.2 \\
1 & $<-0.2$ & $v \geq 5$ & 0.9 & 0.1 & 0.3 & 0.3 & 0.7 & 0.5 & 0.6 & 0.5 \\
1 & $>0.2$ & $v<5$ & 0.9 & 0.1 & 0.3 & 0.3 & 0.7 & 0.5 & 0.6 & 0.5 \\
1 & $>0.2$ & $v \geq 5$ & 0.9 & 0.1 & 0.3 & 0.3 & 0.7 & 0.5 & 0.6 & 0.5 \\
\hline
\end{tabular}

In table IV, $s w l=1$ represents the vehicle is switching to another lane, otherwise it stays in current lane. EL means this vehicle locates in the same lane as the event, and NEL means otherwise. This table presents the prior knowledge that $V_{0}$ uses in calculating the trust of each report by the Bayes' rule.

In our simulation, each vehicle's event report is: 'this accident exists' and its reported belief. For simplicity, we assume that all neighboring vehicles form the same original belief about the event (as they probably received the same set of evidence), which converges to 1 when they get near to the event site (red curve in Fig. 4). Ideally, they should derive the event belief using our framework by combining their neighbors' reports, but that will be unnecessarily complex as the overall convergence trend will be the same. An honest vehicle will report this belief as it is, while a malicious vehicle reports a constant low belief (blue curve in Fig. 4). The implied effect is,

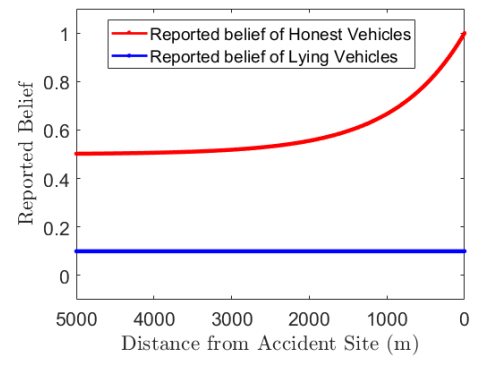

Fig. 4. True and false reported beliefs used in simulation.

if a neighboring vehicle is in the same lane as the accident, they will use our utility function to choose the desired acceleration based on the honest event belief. If they are in a different lane from the reported accident, they will drive at the nominal speed.

All neighboring vehicles may switch lanes with certain possibility corresponding to the prior knowledge in Table II, when they are 2000 meters and 4000 meters away from the accident site. This adds randomness to our simulation to imitate the random behavior of drivers. We use a secure vehicle tracking scheme based on the Kalman Filter (KF) to track the movement of each neighboring vehicle. We assume vehicle $V_{0}$ can obtain a secure distance position measurement of each neighboring vehicle based on radar/lidar/camera [19]-[21] at each time step, which has 0 mean Gaussian noise in $x$ and $y$ directions with variances of $\sigma_{x}^{2}$ and $\sigma_{y}^{2}$ respectively. The initial noise matrix settings are the same as in our previous work [15]. We use the following metrics to measure the robustness and performance of our proposed framework:

(1) Accident-Free Rate (AFR) - the non-crashing percentage of $V_{0}$ averaged over 10,000 Monte Carlo runs for each chosen human factor $h$. A higher AFR demonstrates that a driver will have more confidence that his car is not going to crash.

(2) Potential Crash Speed (PCS) - the average arrival speed $(\mathrm{m} / \mathrm{s})$ of $V_{0}$ at the accident site when it actually exists and meanwhile $V_{0}$ fails to stop. On one hand, a larger arrival speed means a more severe crash if this accident exists. On the other hand, a larger arrival speed is good for the traffic efficiency.

(3) Average Speed (AS) - the average speed $(\mathrm{m} / \mathrm{s})$ of $V_{0}$ during the 5000 meters run. A higher AS means a better transportation efficiency.

\section{A. Attack-resilience against Dishonest Neighboring Vehicles}

We demonstrate the resilience of our framework under different number of dishonest vehicles. We assume $\sigma_{x}=0.1 \mathrm{~m}$ and $\sigma_{y}=1 \mathrm{~m}$. The position error in $x$ direction is relatively small since we showed that it is relatively easier to guarantee accurate localization in $x$ direction [15]. Simulation results are shown in Table V.

In table $\mathrm{V}, N$ is the number of lying vehicles inside this local VANET. When $A F R=1$, there is no PCS. For example, if the number of lying vehicles is 3 and a driver wants to achieve a 0.999 AFR, they will choose $h \geq 1.651$, and consequently expect an average speed of $22.5 \mathrm{~m} / \mathrm{s}$ during the following 5,000 meters. In general, we can see that, with the increase of 
TABLE V

$h$, PCS AND AS UNDER DIFFERENT LEVEL OF AFRS

\begin{tabular}{|c|c|c|c|c|c|c|c|c|c|}
\hline \multirow{2}{*}{$N$} & \multicolumn{2}{|c|}{ AFR $=0.9$} & \multicolumn{2}{|c|}{$\mathrm{AFR}=0.99$} & \multicolumn{2}{|c|}{$\mathrm{AFR}=0.999$} & \multicolumn{2}{|c|}{$\mathrm{AFR}=0.9999$} & AFR=1 \\
\hline & $h$ & PCS AS & $h$ & PCS AS & $h$ & PCS AS & & PCS AS & AS \\
\hline 0 & 1.204 & 30.423 .6 & 1.322 & 13.421 .3 & 1.328 & 13.021 .3 & 1.330 & 13.021 .2 & 1.33121 .2 \\
\hline 1 & 1.287 & 21.523 .5 & 1.444 & 12.821 .1 & 1.451 & 12.721 .0 & 1.453 & 20.9 & 1.45520 .9 \\
\hline 2 & 1.446 & 13.922 .7 & 1.653 & 21.0 & 1.659 & 20.8 & 1.661 & 20.7 & 1.66220 .7 \\
\hline 3 & 1.651 & 12.122 .5 & 1.772 & 11.620 .8 & 1.783 & 11.420 .6 & 1.786 & 11.420 .5 & 1.79020 .5 \\
\hline 4 & 1.994 & 22.0 & 2.27 & 20.5 & 2.28 & 20.3 & 2.25 & 0.920 .2 & 20.2 \\
\hline 5 & 2.631 & 9.920 .9 & 2.893 & 7.219 .5 & 2.912 & $\begin{array}{ll}6.9 & 19.3\end{array}$ & 2.920 & $\begin{array}{ll}6.9 & 19.2\end{array}$ & 2.92419 .2 \\
\hline 6 & 3.543 & 8.920 .4 & 4.021 & $4.9 \quad 18.6$ & 4.074 & $4.7 \quad 18.4$ & 4.091 & $\begin{array}{ll}4.7 & 18.3\end{array}$ & 4.09918 .3 \\
\hline 7 & 4.884 & $\begin{array}{lll}7.0 & 21.3\end{array}$ & 5.066 & $\begin{array}{ll}6.3 & 20.7\end{array}$ & 5.079 & 6.120 .6 & 5.082 & $\begin{array}{ll}6.0 & 20.5\end{array}$ & 5.08420 .5 \\
\hline 8 & 6.647 & 6.223 .5 & 6.814 & 2.422 .7 & 6.862 & 2.322 .6 & 6.874 & 2.322 .6 & 6.87621 .7 \\
\hline 9 & - & - & - & - & - & - & - & - & 8.47522 .8 \\
\hline
\end{tabular}

lying-vehicle percentage, drivers need to choose a higher $h$ to maintain the same level of AFR since a larger $h$ will emphasize more on safety rather than efficiency. Besides, the AS and PCS will decrease as the $h$ increases when the percentage is fixed since a high $h$ represents a more risk-aversion driver, who is willing to sacrifice some speed to avoid danger. Note that, when a large percentage of lying vehicles occurs (i.e. over $80 \%$ ), the average speed of $V_{O}$ increases. This is because the fusion result will be dominated by the false reports which claim 'no accident ahead', and therefore $V_{O}$ keeps running at a relatively high speed even if $h$ is higher. When all vehicles are dishonest, the final event belief will be fixed at 0.1 all the way. Thus, the randomness in the posterior event belief will be eliminated and AFR could only be 0 or 1 for a given $h$. To apply this result in the real-world, we can determine the dishonest vehicle percentage by observing the trust of each vehicle's report, the driver can then choose a proper $h$ according to his own risktaking willingness by referring to the given results.

\section{B. Error Tolerance of Proposed Framework}

In the previous simulation, we set a relatively low measurement error in $y$ direction. However, if $V_{O}$ 's GPS system is under malicious attack, the measurement error may increase and consequently, affect the performance of our system. We next study the impact of measurement error on the system performance. In Figs. 5-7, we compare the AFR, PCS and AS under different $\sigma_{y}$ and $h$ under 3 dishonest neighbor vehicles.

From Fig. 5, as the position measurement error increases, the AFR decreases under the same $h$, because a larger measurement error affects the decision accuracy. Figs. 6 and 7 show that the PCS and AS would keep constant under the same $h$ for different levels of noise since the mean of the measurement error is 0 . In other words, when the noise level increases, the AS and PCS will decrease if drivers want to achieve the same AFR as the corresponding $h$ increases. In general, a larger noise will lead to worse efficiency under the same safety level.

\section{Performance Comparison of Different Combination Rules}

Next, we will compare the Han's combination rule with the Weighted DST (WDST) as well as the Majority Voting (MV) to demonstrate the superiority of our data fusion approach.
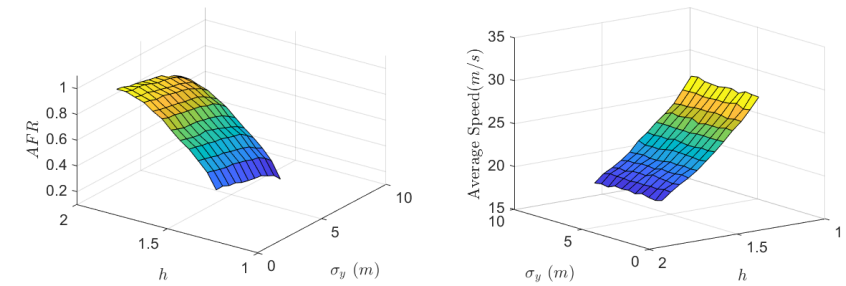

Fig. 5. AFR under different measure-Fig. 6. AS under different measurement accuracy and $h \quad$ ment accuracy and $h$

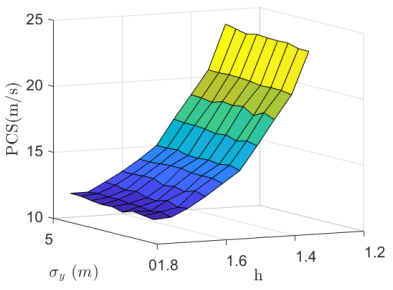

Fig. 7. PCS under different measurement accuracy and $h$

In this simulation, we assume that $\sigma_{y}=1 \mathrm{~m}$ and there are 7 neighbors lying (dishonest majority) in this local VANET. The performance of different combination rules is listed in Table VI.

TABLE VI

$h$, PCS AND AS UNDER MV, WDST AND HAN'S RULE

\begin{tabular}{cccccccccccccc}
\hline \multirow{2}{*}{ Rule name } & \multicolumn{3}{c}{ AFR=0.9 } & \multicolumn{4}{c}{ AFR=0.99 } & \multicolumn{3}{c}{ AFR=0.999 } & \multicolumn{3}{c}{ AFR=1 } \\
& $h$ & PCS & AS & $h$ & PCS & AS & $h$ & PCS & AS & $h$ & AS \\
\hline Han's & 4.884 & 7.0 & 21.3 & 5.066 & 6.3 & 20.7 & 5.079 & 6.1 & 20.6 & 5.084 & 20.5 \\
WDST & 5.794 & 5.4 & 11.7 & 6.012 & 4.8 & 11.4 & 6.045 & 4.6 & 11.3 & 6.047 & 11.3 \\
Raya [10] & 8.367 & 4.3 & 9.7 & 8.982 & 3.6 & 9.4 & 9.045 & 3.5 & 9.4 & 9.059 & 9.4 \\
MV & 11.134 & 3.5 & 8.3 & 11.866 & 3.2 & 8.0 & 11.875 & 3.1 & 7.9 & 11.879 & 7.9 \\
\hline
\end{tabular}

The table VI shows that the Han's combination rule achieves better efficiency under the same safety level than WDST and MV. We have to set a very large $h$ to compensate for the majority voting scheme because it always outputs the totally wrong event belief, which consequently leads to lower AS and PCS. As for the WDST, since it is a conservative rule, the AS will drop compared with Han's rule under same AFR, which means a lower efficiency for our vehicle. Raya's work [10] adopted the Dempster's rule to evaluate the trust and its performance is better than MV but worse than WDST under the same safety level when combined with our utility scheme.

\section{CONCLUSION AND Future Work}

This paper presents a novel truth-aware decision-making framework in V2V communications, that examine the existence of a reported event and make an optimal vehicle response under uncertain and conflicting/fake data. Our framework is based on the idea of verifying the implied effect of one road events, which is achieved by using the Bayes' rule and DST to estimate the trustworthiness of each vehicle report and fuse them together. Besides, we incorporate driver preferences in the optimal response to achieve a desired balance between safety and efficiency. As simulation results show, our framework achieves high AFR under a dishonest majority and different 
noise levels. It also provides guidance for drivers to choose appropriate human preferences. In the future, we will extend this framework to handle multi-hop forwarding and belief combination. Besides, we will explore the use of dynamic decision network to further optimize the decision-making.

\section{ACKNOWLEDGEMENT}

This work was partly supported by NSF grants CNS1410000 and CNS-1619728.

\section{REFERENCES}

[1] D. Jiang and L. Delgrossi, "Towards an international standard for wireless access in vehicular environments," in VTC Spring. IEEE, 2008, pp. 2036-2040.

[2] G. S. Tewolde, "Sensor and network technology for intelligent transportation systems," in Electro/Information Technology (EIT), 2012 IEEE International Conference on. IEEE, 2012, pp. 1-7.

[3] R. Hussain, J. Son, H. Eun, and Kim, "Rethinking vehicular communications: Merging vanet with cloud computing," in CloudCom, IEEE 4th International Conference. IEEE, 2012.

[4] S. Sheikholeslam and C. A. Desoer, "Longitudinal control of a platoon of vehicles," in American Control Conference, 1990. IEEE, 1990.

[5] Yibada, "Waze implements safeguards to prevent 'ghost cars'," 2016.

[6] T. Jeske, "Floating car data from smartphones: What google and waze know about you and how hackers can control traffic," 2013. [Online]. Available: https://media.blackhat.com/eu-13/briefings/Jeske,2013

[7] N. Tufnell, "Students hack waze, send in army of traffic bots," 2014. [Online]. Available: http://www.wired.co.uk/article/ waze-hacked-fake-traffic-jam

[8] M. Raya and J.-P. Hubaux, "Securing vehicular ad hoc networks," Journal of Computer Security, vol. 15, no. 1, pp. 39-68, 2007.

[9] M. Gerlach, "Trust for vehicular applications," in ISADS. IEEE, 2007, pp. 295-304.

[10] M. Raya and P. Papadimitratos, "On data-centric trust establishment in ephemeral ad hoc networks," in INFOCOM. IEEE, 2008.

[11] X. Wang, "Trust and independence aware decision fusion in distributed networks," in IEEE PERCOM Workshops. IEEE, 2013, pp. 481-486.

[12] N. Bißmeyer, "Central misbehavior evaluation for vanets based on mobility data plausibility," in Proc. of the 9th workshop on Vehicular inter-networking, systems and Apps. ACM, 2012, pp. 73-82.

[13] M. Ghosh, A. Varghese, A. A. Kherani, and A. Gupta, "Distributed misbehavior detection in vanets," in Proceedings of IEEE WCNC. USA: IEEE Press, 2009, pp. 2909-2914.

[14] Z. Wei, F. R. Yu, and A. Boukerche, "Trust based security enhancements for vehicular ad hocnetworks," in Proceedings of the fourth ACM international symposium on Development and analysis of intelligent vehicular networks and applications. ACM, 2014, pp. 103-109.

[15] M. Sun, M. Li, and R. Gerdes, "A data trust framework for vanets enabling false data detection and secure vehicle tracking," in 2017 IEEE CNS), Oct 2017, pp. 1-9.

[16] R. Coussement, B. Amar Bensaber, and I. Biskri, "Decision support protocol for intrusion detection in vanets," in Proceedings of the third ACM international symposium on Design and analysis of intelligent vehicular networks and applications. ACM, 2013, pp. 31-38.

[17] J. Zhang, C. Chen, and R. Cohen, "Trust modeling for message relay control and local action decision making in vanets," Security and Communication Networks, vol. 6, no. 1, pp. 1-14, 2013.

[18] Q. Xu, T. Mak, J. Ko, and R. Sengupta, "Vehicle-to-vehicle safety messaging in dsrc," in ACM VANET. ACM, 2004, pp. 19-28.

[19] T. Tithi, C. Winstead, and R. Gerdes, "Viability of using shadows cast by vehicles for position verification in vehicle platooning," in Trustcom/BigDataSE/ICESS, 2017 IEEE. IEEE, 2017, pp. 210-217.

[20] S. Mitchell and R. M. Gerdes, "Visual distance estimation for pure pursuit based platooning with a monocular camera," in ACC, 2017. IEEE, 2017, pp. 2327-2332.

[21] E. Yeh and J. Choi, "Security in automotive radar and vehicular networks," submitted to Microwave Journal, 2016.

[22] J. Burge, "Decision-aid system based on wirelessly-transmitted vehicle crash sensor information,’ Jul. 16 2001, uS Patent App. 09/907,542.
[23] D. Han, C. Han, and Y. Yang, "A modified evidence combination approach based on ambiguity measure," in Information Fusion, 2008 11th International Conference on. IEEE, 2008, pp. 1-6.

[24] L. A. Zadeh, "A simple view of the dempster-shafer theory of evidence and its implication for the rule of combination," AI magazine, vol. 7, no. 2, p. 85, 1986.

[25] N. L. Zhang, R. Qi, and D. Poole, "A computational theory of decision networks," International Journal of Approximate Reasoning, vol. 11, no. 2, pp. 83-158, 1994.

[26] H. Wu, M. Siegel, R. Stiefelhagen, and J. Yang, "Sensor fusion using dempster-shafer theory [for context-aware hci]," in Instrumentation and Measurement Technology Conference, 2002. IMTC/2002. Proceedings of the 19th IEEE, vol. 1. IEEE, 2002, pp. 7-12.

[27] P. C. Fishburn, "Utility theory for decision making," Research Analysis Corp Mclean va, Tech. Rep., 1970.

[28] P. L. Brockett and L. L. Golden, "A class of utility functions containing all the common utility functions," Management Science, vol. 33, no. 8, pp. 955-964, 1987.

[29] J. R. Adams-Guppy and A. Guppy, "Speeding in relation to perceptions of risk, utility and driving style by british company car drivers," $E r$ gonomics, vol. 38, no. 12, pp. 2525-2535, 1995.

[30] H. Ben-Zur and M. Zeidner, "Threat to life and risk-taking behaviors: A review of empirical findings and explanatory models," Personality and Social Psychology Review, vol. 13, no. 2, pp. 109-128, 2009.

[31] E. Jacquet-Lagrèze and J. Siskos, "Assessing a set of additive utility functions for multicriteria decision-making, the uta method," European journal of operational research, vol. 10, no. 2, pp. 151-164, 1982.

[32] M. S. Bazaraa, Nonlinear programming: theory and algorithms. John Wiley \& Sons, 2013

[33] IIHSHLDI, "Vehicle accident report," 2016. [Online]. Available: http://www.iihs.org/iihs/topics/t/general-statistics/fatalityfacts

\section{APPENDIX}

For the single event case, an evidence only have two focal elements which are event "happen (A)" or "not happen (B)". Also, $m(A)+m(B)=1$. If we have two pieces of evidence $m_{1}$ and $m_{2}$ with normalize weight $w_{1}$ and $w_{2}$, we can rewrite the above expressions as:

$$
\begin{aligned}
& m_{12}(A)=m_{1}(A) m_{2}(A)+w_{1} m_{1}(A) m_{2}(B)+w_{2} m_{1}(B) m_{2}(A) \\
& =m_{1}(A) m_{2}(A)+w_{1} m_{1}(A)\left(1-m_{2}(A)\right)+w_{2}\left(1-m_{1}(A)\right) m_{2}(A) \\
& =w_{1} m_{1}(A)+w_{2} m_{2}(A)
\end{aligned}
$$

For three sources, we combine them and obtain the $m_{123}(A)$ :

$$
\begin{aligned}
& m_{123}(A)=m_{12}(A) m_{3}(A)+\frac{w_{1}+w_{2}}{w_{1}+w_{2}+w_{3}} m_{12}(A)\left(1-m_{3}(A)\right) \\
& +\frac{w_{3}}{w_{1}+w_{2}+w_{3}} m_{3}(A)\left(1-m_{12}(A)\right) \\
& \left.=m_{12}(A) m_{3}(A)\right)-\left(w_{1}+w_{2}\right) m_{12}(A) m_{3}(A)-w_{3} m_{12}(A) m_{3}(A) \\
& +\left(w_{1}+w_{2}\right) m_{12}(A)+w_{3} m_{3}(A) \\
& =\left(w_{1}+w_{2}\right) m_{12}(A)+w_{3} m_{3}(A)
\end{aligned}
$$

where $m_{12}(A)=\frac{w_{1}}{w_{1}+w_{2}} m_{1}(A)+\frac{w_{2}}{w_{1}+w_{2}} m_{2}(A) \cdot w_{1}, w_{2}, w_{3}$ are the normalized weight for $m_{1}, m_{2}$ and $m_{3}$ respectively. Then we can get the final results:

$$
m_{123}(A)=w_{1} \cdot m_{1}(A)+w_{2} \cdot m_{2}(A)+w_{3} \cdot m_{3}(A)
$$

In conclusion, for three sources of evidence, the combination results is a weighted average for the single event evidence. The above can also be extended to multiple-source cases. 